\title{
PTD-J domain acts as an antigen carrier for cell mediated immunity
}

Tai-Yun Lin, Yu-Hsiu Su and Chin-kai Chuang*

*Correspondence: jkjuang@mail.atri.org.tw

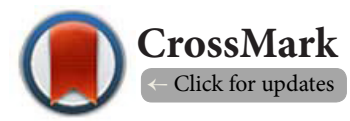

Division of Animal Technology, Laboratories of Animal Technology, Agricultural Technology Research Institute No. 1, Lane 51, Dahu Rd., Xiangshan District, Hsinchu City 30093, Taiwan.

\begin{abstract}
BALB/c mice elicited specific cell-mediated immunity after transplantation with CT26 cells stably expressing human telomerase reverse transcriptase (CT26(hTERT\#10)). Restingmemory T cells were reactivated in vitro by CT26(hTERT\#10) cells and PTD-J-hTERTepi recombinant proteins, in which the polypeptide hTERTepi comprised TH and TC epitopes, as indicated by interferon $\gamma$ (IFN $\gamma$ ) secretion. Preand post-injections of PTD-J-hTERTepi with in vitro expanded and differentiated dendritic cells reduced CT26(hTERT\#10) tumor size. Moreover, Hsp72 activated numerous splenocytes to perform IFN $\gamma$ secretion and further reduced tumor sizes.
\end{abstract}

Keywords: PTD-J domain, hTERT, epitopes, cellular immunity

\section{Introduction}

hTERT is a ubiquitous candidate for cancer immunotherapy Human telomerase reverse transcriptase (hTERT) is the catalytic subunit of human telomerase and is reportedly expressed in $85 \%$ of human malignant tumors, but not in normal somatic tissues [1-3]. Hence, telomeres and telomerase are potential targets of cancer therapy $[4,5]$, and hTERT has emerged as the first bona fide common tumor antigen $[6,7]$ and is actively investigated as a target for cancer immunotherapy [8]. Although hTERT is rapidly degraded in cancer cells and the resulting peptides are presented on de novo synthesized major histocompatibility complex class I (MHC I) molecules, the resulting signals lack sufficient intensity to elicit cytotoxic lymphocyte (CTL) responses with assistance from specific CD4+T helper 1 $\left(T_{H} 1\right)$ cells. MHC I molecules load peptides in the endoplasmic reticulum (ER) and display them for T cell receptors $(T C R)$ on CD8+ T lymphocytes ( $\mathrm{T}_{\mathrm{C}}$ ). MHC class II molecules (MHCII) acquire peptides from endosomes and present these complexes to TCR on CD4+T lymphocytes. Among adaptive immune cells involved in anti-tumor responses, $T_{C}$ cells have been considered as the main protagonists because they elicit direct cytotoxic activity toward cancer cells. However, tumor reactive $T_{H} 1$ cells that produce IFNY, TNF $\alpha$, and IL-2 play critical roles in the orchestration of cell-mediated immunity against tumors [9].

\section{PTD-J-X recombinant protein behaves as both intracellular} and extracellular antigen

As fore mentioned, intracellular antigens are processed and primed onto $\mathrm{MHCl}$ in the $\mathrm{ER}$, whereas extracellular antigens are processed and presented on endosomal MHC II. Recombinant proteins are usually considered as extracellular antigens; however cell penetrating peptides and their cargos can traverse the cytoplasm membrane $[10,11]$ and can be considered as intra cellular antigens. Recently, we reported an expression system for are combinant PTD-J-cargo protein comprising a protein transduction domain (PTD), a J domain of Hsp40, and a cargo polypeptide [12]. Subsequently, we showed that the cargo polypeptide assembly displayed epitopes of FMDV VP1 protein fused with PTD-J and elicited humoral immunity against VP1and Hsp72 which is able to associate with the J domain can enhance the humoral immunogenicity of the cargo of PTD-J-cargo [13]. Since we had proven that the cell penetrating activity of PTD-J-DsRed was stronger than that of PTD-DsRed [14]. It was interesting to test whether the PTDJ-cargopolypeptide could be used as intracellular antigen to elicit cellular immunity or not. The PTD-J-hTERTepi, where the cargo polypeptide hTERTepi is composed of MHC I and MHC II binding peptides of hTERT, was expressed and utilized to verify this idea. The ectopic Hsp72 recombinant protein was also supplemented to test whether it could enhance cellular immunity or not. Moreover, bone marrow derived syngeneic dendritic cells were used to present the antigen.

\section{Materials and methods}

Stable expression of hTERT in CT26 cells

The hTERT-Flag ${ }_{2}$-IREShyg expression cassette was separated 
Lin et al. Journal of Immunotherapy Applications 2015,

http://www.hoajonline.com/journals/pdf/2055-2394-2-1.pdf

doi: 10.7243/2055-2394-2-1

from the pCX-hTERT-Flag ${ }_{2}$-IREShyg vector (Supplementary Figure S1) using Not I digestion and was transfected into CT26 colon carcinoma cells. Cell colonies resistant to $400 \mu \mathrm{g} /$ $\mathrm{mL}$ hygromycin $\mathrm{B}$ were picked and expanded in the presence of $200 \mu \mathrm{g} / \mathrm{mL}$ hygromycin B. Selected cells expressing recombinant hTERT-Flag ${ }_{2}$ were cultured without hygromycin B for one week and recombinant protein expression was analyzed using western blotting. Cell lines with stable expression of recombinant hTERT-Flag ${ }_{2}$ (such as CT26(hTERT \#10)), and those that stopped expressing it (such as CT26(hTERT \#4)) in the absence of hygromycin B were collected.

\section{Preparation of the pET22b-PTD-J-hTERTepi expression vector}

Published hTERT peptides associated with various well characterized human class I and class II MHC alleles $[\mathbf{8 , 1 5 , 1 6 ]}$ and predicted hTERT epitopes of class I and class II MHC from the mouse $\mathrm{H}-2^{\mathrm{d}}$ locus were identified using neural network [17] and stabilized matrix methods [18] (Figure 1a). According to these data, hTERT $_{602-641}$ (SEAEVRQHREARPALLTSRLRFIPKPDGLRPIVNMDYVVG) and hTERT ${ }_{1088-1121}$ (TYVPLLGSLRTAQTQLSRKLPGTTLTALEAAANP) were selected and linked by an FFRK peptide to produce hTERTepi. Corresponding CDNA with E. coli optimized codons was synthesized as shown in Supplementary Figure S2 and inserted into a pET22b-PTD $J_{1}(\mathrm{~Tb})$ vector [12] (Supplementary Figure S3) between EcoRI and Xhol sites to produce the pET22b-PTD-J-hTERTepi expression vector.

\section{Expression and purification of the PTD-J-hTERTepi recombinant protein}

E. coli Rosetta-gamiB (DE3)pLysSRARE cells carrying pET22bPTD-J-hTERTepi were cultured overnight in LB supplemented with $30 \mu \mathrm{g} / \mathrm{mL}$ carbenicillin and $25 \mu \mathrm{g} / \mathrm{mL}$ chloramphenicol, and were then centrifuged at $5,000 \mathrm{~g}$ for $10 \mathrm{~min}$. Cell pellets were suspended in five volumes of $2 \times Y$ T medium supplemented with $0.4 \%$ glucose and $30 \mu \mathrm{g} / \mathrm{mL}$ carbenicillin, and were cultured for about $2 \mathrm{~h}$ until the $\mathrm{OD}_{600}$ value was around 1. IPTG concentrations were then adjusted to $1 \mathrm{mM}$ to induce recombinant protein expression for $4 \mathrm{~h}$. Subsequently, PTD-J-hTERTepi recombinant protein was expressed in an insoluble inclusion body form, which was then separated from soluble lysates by centrifugation at $20,000 \mathrm{~g}$ for $20 \mathrm{~min}$. The pellet was then dissolved in a buffer containing $50 \mathrm{mMTris}-\mathrm{HCl}, 300 \mathrm{mMNaCl}$, and $6 \mathrm{M}$ guanidine $-\mathrm{HCl}(\mathrm{pH} 8.0)$, and insoluble debris was removed by centrifugation at $20,000 \mathrm{~g}$ for $30 \mathrm{~min}$. The supernatant fraction was applied to a Ni-NTA sepharose (fast flow, GE Healthcare) column with a flow rate of 5 column volumes per hour. The column was then washed with 10 column volumes of detergent buffer containing $20 \mathrm{mMTris}-\mathrm{HCl},(\mathrm{pH} 8.0)$, $100 \mathrm{mMNaCl}, 1 \%$ Triton X-100, and 10mM 2-mercaptoethanol. Detergents were stripped from recombinant protein by washing with 10 column volumes of cyclodextrin buffer containing $20 \mathrm{mMTris}-\mathrm{HCl}(\mathrm{pH} 8.0), 100 \mathrm{mMNaCl}$, and $5 \mathrm{mM} \beta$-cyclodextrin. After washing the column with 10 column volumes of wash

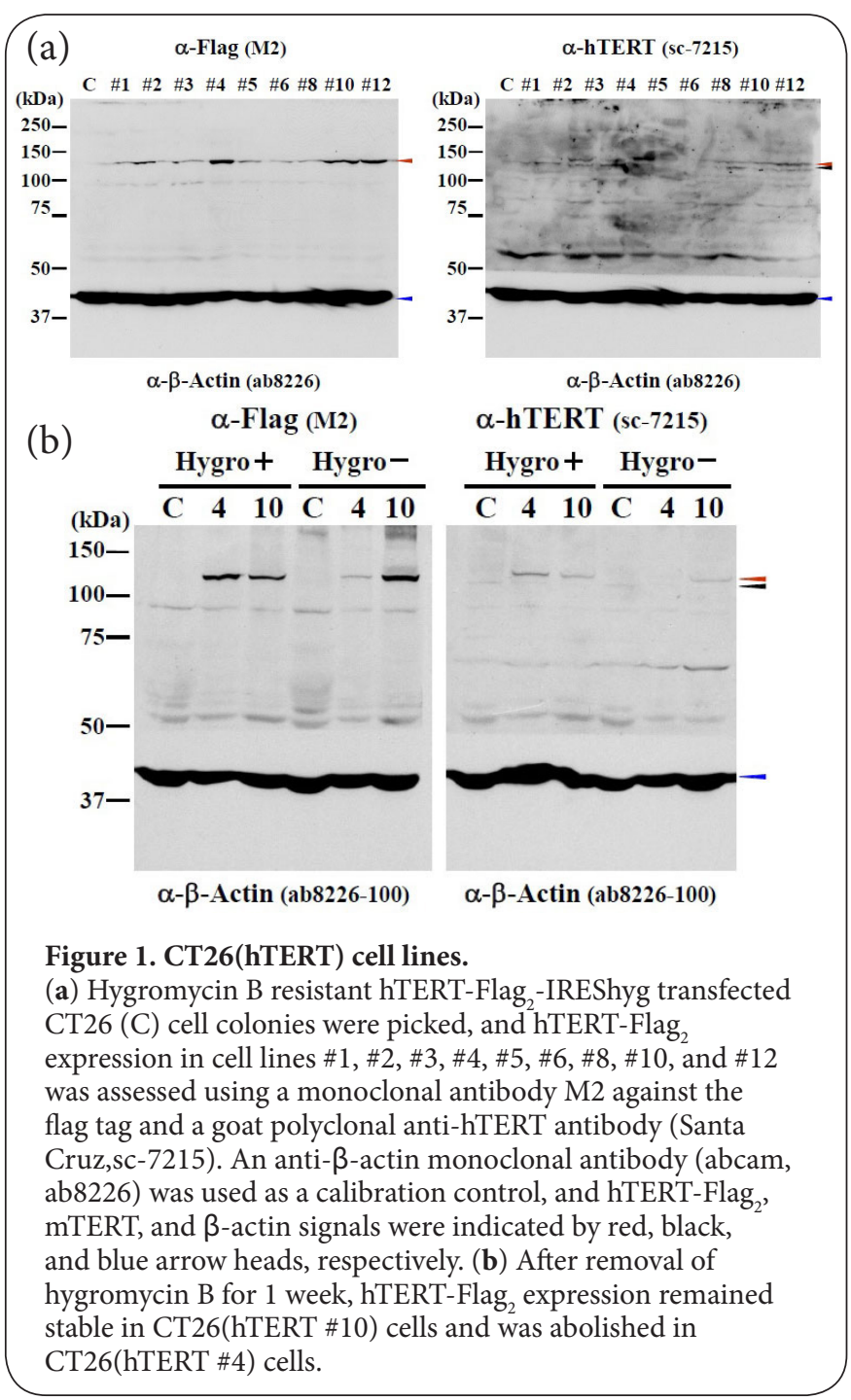

buffer containing $20 \mathrm{mMTris}-\mathrm{HCl}(\mathrm{pH} 8.0)$ and $500 \mathrm{mMNaCl}$, the recombinant protein was eluted in anelution buffer containing $20 \mathrm{mMTris}-\mathrm{HCl}(\mathrm{pH} 7.4), 300 \mathrm{mMNaCl}$, and $250 \mathrm{mM}$ imidazole.

\section{Transplantation of cancer cells into BALB/c mice}

CT26, CT26 (hTERT\#10), and CT26 (hTERT\#4) cells $\left(1 \times 10^{6}\right)$ were implanted subcutaneously into dorsal-lateral sides of 5 sets, three mice per set, of BALB/c mice (Figure 2). Sets one, two, and three received CT26, CT26 (hTERT \#10), and CT26 (hTERT \#4) cell implants into both sides, respectively (sets I-III), and sets four and five received cell implants of CT26 and CT26 (hTERT\#10) (set IV), and CT26 and CT26 (hTERT\#4) (set V) cells into either side, respectively. Ten days after cancer cell implantation, long (L) and short $(\mathrm{S})$ diameters were measured twice a week using a caliper and tumor volumes were estimated as $1 / 2 L^{2}$.

\section{Immunization of $B A L B / c$ mice}

Bone marrow derived dendritic cells (DCs) were prepared from 


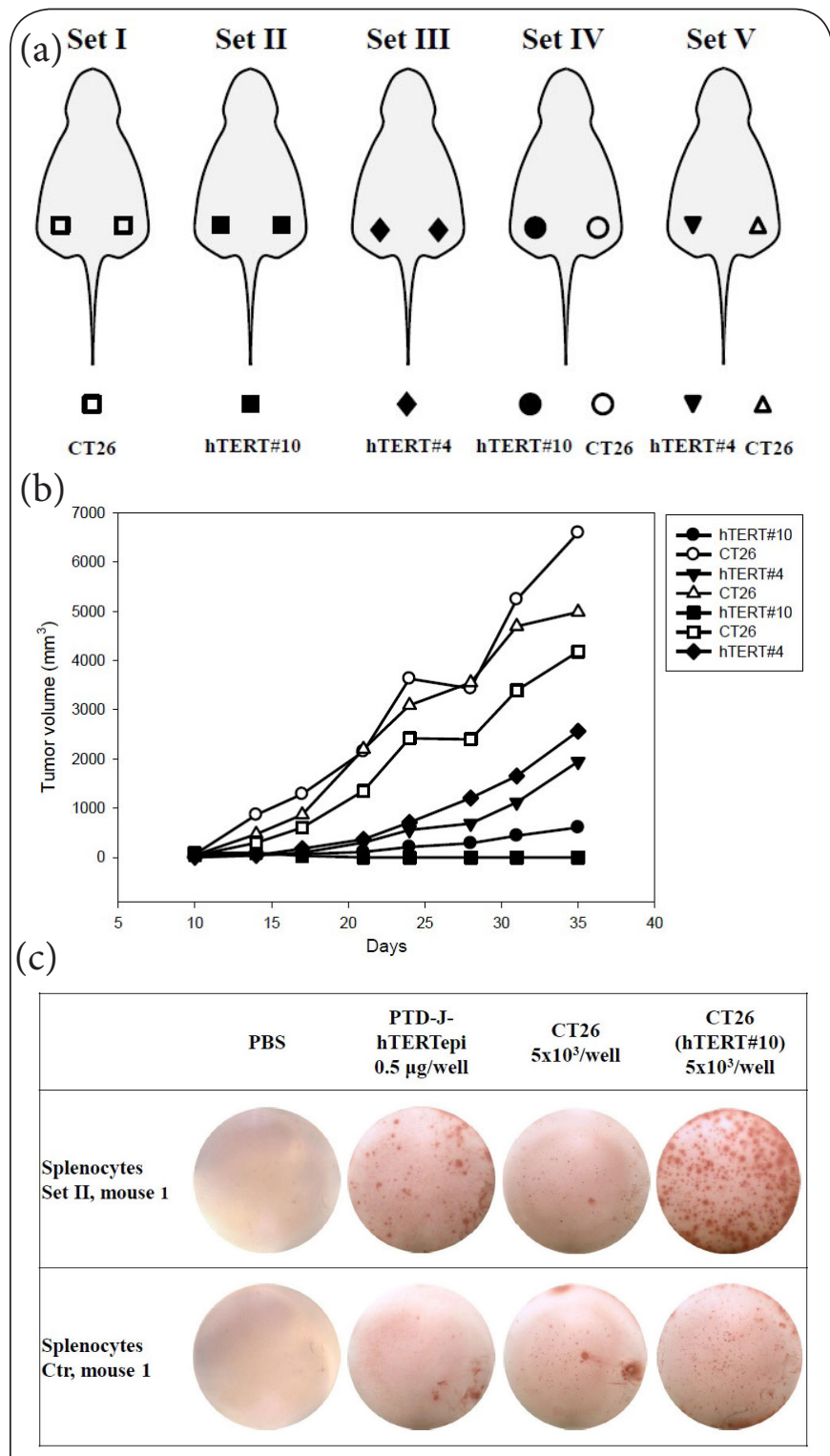

Figure 2. Transplantation of CT26,CT26(hTERT\#10), and CT26(hTERT\#4) cell lines into BALB/c mice.

(a) Five sets of mice, three mice per set, received surgery. CT26, CT26(hTERT \#10), and CT26(hTERT \#4) cells were injected subcutaneously into both dorsal and lateral sides (set I, set II, and set III respectively), CT26 and CT26(hTERT\#10) cells were injected into either side (set IV), and CT26 and CT26(hTERT\#4) cells were injected into either side (set V). (b) Long (L) and short diameters (S) were measured twice a week from 10 days after cancer cell implantation using a caliper and tumor volumes were estimated as $1 / 2 \mathrm{LS}^{2}$. (c) Two months after the experiment, RBC-depleted splenocytes were collected from the second set of mice and from untreated age-matched control mice. T cells with memories of hTERT epitopes were evoked by treatment withPTD-J-hTERTepi or CT26(hTERT\#10) for $24 \mathrm{~h}$.

8-week-old BALB/c mice as previously described [19]. Briefly, DC progenitor cells were expanded in RPMI1640 medium containing $10 \% \mathrm{FCS}, 20 \mathrm{ng} / \mathrm{mL}$ recombinant murine Flt3L, $10 \mathrm{ng} / \mathrm{mL}$ recombinant murine $\mathrm{IL}-3,10 \mathrm{ng} / \mathrm{mL}$ recombinant murine IL-6, and $10 \mathrm{ng} / \mathrm{mL}$ recombinant murine SCF for 18 days. Subsequently, progenitor cells were differentiated using 20ng/ $\mathrm{mL}$ IL-4 and $20 \mathrm{ng} / \mathrm{mL}$ GM-CSF for 5 days, and $1 \times 10^{6}$ differentiated isolated DCs mixed with $35 \mu \mathrm{g}$ of PTD-J-hTERTepi or with $35 \mu \mathrm{g}$ of PTD-J-hTERTepi and $137 \mu \mathrm{g}$ of recombinant murine Hsp72 [12] in $0.5 \mathrm{~mL}$ of PBS were injected intraperitoneally into 6-week-old BALB/c mice. At 7 days after CT26(hTERT\#10) implantation, $\mathrm{L}$ and $\mathrm{S}$ diameters were measured every fifth day as aforementioned.

\section{IFN $\gamma$ ELISPOT assay}

PVDF membranes of ELISPOT plates' (Millopore, Cat. No., MAIPS-4510) wells were coated with $0.25 \mu \mathrm{g}$ of rat anti-mouse interferony (clone R4-6A2, BioLegend, Cat. No. 505701) for more than $4 \mathrm{~h}$ at $4^{\circ} \mathrm{C}$. After blocking, $1 \times 10^{6} \mathrm{RBC}$-depleted splenocytes (response cells) with $0.5 \mu \mathrm{g}$ of PTD-J-hTERTepi, $5 \times 10^{3} \mathrm{CT} 26$, or $5 \times 10^{3} \mathrm{CT} 26$ (hTERT\#10) cells (stimulators) were added to each well and cultured for $24 \mathrm{~h}$. Cells were removed by washing three times with PBS and then 3 times with PBS containing $0.05 \%$ Tween-20. Subsequently, $0.1 \mu \mathrm{g}$ of biotinylated rat antimouse monoclonal antibody clone XMG1.2 (BioLegend, Cat. No. 505803) in PBS containing 0.05\% Tween-20 was applied to each well and incubated for $2 \mathrm{~h}$ at room temperature. After four washes with PBS containing $0.05 \%$ Tween-20, $100 \mu \mathrm{L}$ of a 1:100 dilution of avidin-HRP (BD Biosciences, Cat No. 557630) was added to each well and incubated for $1 \mathrm{~h}$ at room temperature. The plate was then washed three times with PBS containing $0.05 \%$ Tween-20. Finally, $100 \mu \mathrm{L}$ of AEC substrate (BD Biosciences, Cat No. 551951) was added to each well and spots were developed. The color forming reaction was stopped by rinsing the plates with tap water, and spots were observed under a dissecting microscope after air drying the plates.

\section{Results}

$\mathrm{BALB} / \mathrm{c}$ mice recognized hTERT epitopes on syngenic CT26 cells

After transfection with the hTERT-Flag ${ }_{2}$-IREShyg gene expression cassette, CT26 derived cell lines were selected under $400 \mu \mathrm{g} / \mathrm{mL}$ hygromycin B. Among these cell lines, CT26(hTERT\#4), CT26(hTERT\#10), and CT26(hTERT\#12) cells expressed higher levels of recombinant hTERTFlag $_{2}$ (Figure 1a). The hTERT-Flag 2 recombinant protein expression level would be abolished in CT26(hTERT\#4) after the removal of hygromycin B selection, whereas CT26(hTERT\#10) cells expressed it consistently (Figure 1b). Besides the hTERT-Flag 2 protein, endogenous mouseTERT (mTERT) was expressed in all the CT26 cell lines (Figure 1a).

Transplanted CT26 cells grow infinitely in syngenic BALB/C mice indicating that $C T 26$ cells can escape from the immune surveillance of the hosts. It is curious that CT-26 expressing xenogenically intracellular hTERT can be recognized by the immune system or not. The CT26(hTERT\#10) which consistently expresses hTERT-Flag ${ }_{2^{\prime}}$ CT26(hTERT\#4) which expresses hTERT-Flag ${ }_{2}$ only under the presence of hygromycin $B$, and 
Lin et al. Journal of Immunotherapy Applications 2015,

CT26 cell lines were chosen to figure out this question. Five sets, three mice per set, of BALB/c mice were implanted subcutaneously into dorsal-lateral sides with $1 \times 10^{6}$ cells per site of CT26, CT26(hTERT\#10), and CT26(hTERT\#4) (Figure 2a). As mentioned above, the CT26 tumors proliferated most quickly. In contrast, CT26(hTERT\#10) tumors grew for about 14 days after transplantation and then receded rapidly, becoming undetectable by 24 days after transplantation. These mice were observed for another two months and no tumor relapse was observed. This result indicated that certain immunity was elicited by CT26(hTERT\#4) cells which escaped growth suppression from the $17^{\text {th }}$ day after transplantation (Figure 2, set I, II and III). In further experiments, CT26 cells were injected into single sides of mice, and the other side was injected with either CT26(hTERT\#10) or CT26(hTERT\#4) cells. CT26 tumor growth rates were not influenced by the presence of CT26(hTERT\#10) or CT26(hTERT\#4) tumors, and relative tumor sizes reflected nutrient competition (Figure 2, set IV and V). In this situation, CT26(hTERT\#10) tumors grew slowly but continuously during the experimental period (Figure 2, set V).

Preparation of the PTD-J-hTERTepi recombinant protein Since hTERT was demonstrated to be an intracellular antigen that elicited cellular immune responses, an immunogenic composition was designed. Based on the published human MHC I and MHC II binding hTERT peptides, and those carrying predicted associations with mouse $\mathrm{MHC}$ I and $\mathrm{MHC}$ II of $\mathrm{H}-2^{\mathrm{d}}$ genotypes summarized in Figure $3 \mathrm{a}$, two fragments hTERT $_{602-641}$ and hTERT ${ }_{1088-1121}$ contained both human and murine candidates and were selected to assemble the multiepitopic hTERTepi polypeptide (Figure 3b). Subsequently, the recombinant PTD-J-hTERT epi protein was expressed by E. coli as an inclusion body, and was refolded and purified (Figure 3c). Very few isolated splenocytes from CT26(hTERT\#10) transplanted mice (Figure $\mathbf{2 b}$ ) were sensitive to CT26 cells at two months after the experiment. However, a certain fraction of splenocytes were invoked by PTD-J-hTERTepi protein or CT26 (hTERT\#10) cells to express IFNY (Figure 2c).

\section{PTD-J-hTERT epi elicits tumor-suppressing activity}

Because membrane penetration of the PTD peptide was enhanced by the J domain [14], bone marrow derived DCs were used to present the processed peptide products of PTD-J-hTERT epi after penetration into the cytoplasm and endocytotic engraftment. Hsp72 was previously shown to augment the immune response [13] and was further examined during two immunization courses. Mice were either immunized at 9 and 2 days before transplantation of CT26 (hTERT\#10) cells or at 2 and 9 days after transplantation, and mice were divided into three subsets for each course. Mice of the first subset were injected intraperitoneally with DCs alone, whereas those of the second subset were injected with DCs and PTD-J-hTERT epi recombinant protein (DCs/PTD-J-hTERTepi), and mice of the third set were treated with $D C$, recombinant
PTD-J-hTERTepi and Hsp72 proteins (DCs/PTD-J-hTERTepi/ Hsp72) in $0.5 \mathrm{~mL}$ of PBS. At 7 days after implantation of CT26 (hTERT\#10) cells, tumor volumes were measured every fifth day using a pair of calipers (Figure 4a). In these experiments, CT26(hTERT\#10) tumor volumes were about $105 \mathrm{~mm}^{3}$ at 12 days after transplantation, but were subsequently diminished by host immune activities thereafter. CT26 (hTERT\#10) tumor sizes in mice that were pre-treated or post-treated with DCs, DCs/PTD-J-hTERTepi, or DCs/PTD-J-hTERTepi and Hsp72 were significantly smaller than those of untreated mice. Comparisons of tumor volumes on the $17^{\text {th }}$ day (Figure $4 \mathbf{b}$ ) indicated that injection of in vitro expanded and differentiated DCs from autologous bone marrow retards tumor growth. Moreover injections of DCs with recombinant PTD-J-hTERTepi protein further limited CT26 (hTERT\#10) tumor growth, whereas the addition of Hsp72 to DCs/PTD-J-hTERTepi had marginal effect on the reduction of tumor volumes.

\section{Injection of PTD-J-hTERTepi and Hsp72 with DCs increases numbers IFN $\gamma$-expressing splencytes}

Both pre- and post-injections of DCs with recombinant PTD-J-hTERTepi could further reduce CT26(hTERT\#10) tumor volumes than treated with DCs alone. These results indicated that cytotoxic immunity might be elicited by PTD-J-hTERTepi. This cytotoxic immunity might be augmented by Hsp72. ELISPOT experiments were performed to determine numbers of IFNy-expressing splenocytes from RBC-depleted spleen at 7 days after two successive injections of PBS, DCs, DCs/PTD-JhTERTepi or DCs/PTD-J-hTERTepi/Hsp72. These experiments showed very few IFN $\gamma$ secreting splenocytes $\left(9\right.$ in $\left.10^{6}\right)$ from the DCs injected group in comparison with PBS treated controls. The numbers of IFNY secreting splenocytes after injection of DCs/PTD-J-hTERTepi increased significantly (about 510 in $10^{6}$ ), and out of scale numbers (more than 3000 in $10^{6}$ ) were illustrated in the DCs/PTD-J-hTERTepi/Hsp72 cases (Figure 5).

\section{Discussion}

$\mathrm{BALB} / \mathrm{c}$ mice specifically recognized xenogenic hTERT antigen expression in syngeneic CT26 cancer cells

Syngeneic CT26 colorectal cancer cells escaped immune surveillance in BALB/c mice, resulting in tumor growth (Figure 2, set I), whereas CT26(hTERT\#10) cells were eliminated by immune activities (Figure 2, set II). However, after transplantation of CT26 and CT26(hTERT\#10) cells at separate sites in the same mice, CT26 tumor formation was similar, even a little faster, to that in the mice of set l, and CT26 (hTERT\#10) tumors grew very slowly (Figure 2, set IV). The CT26 (hTERT\#10) tumors in set II were completely eliminated by 24 days, however, those in set IV were not. It revealed that the accompany CT26 tumors in set IV mice could suppress the immunity of the hosts. These results indicate that intracellular xenogenic hTERT expression in CT26 cells elicited immune responses in BALB/c mice. Moreover, about 230 of $10^{6}$ splenocytes from set II mice were reactivated at two months after transplantation with $\mathrm{CT} 26$ 


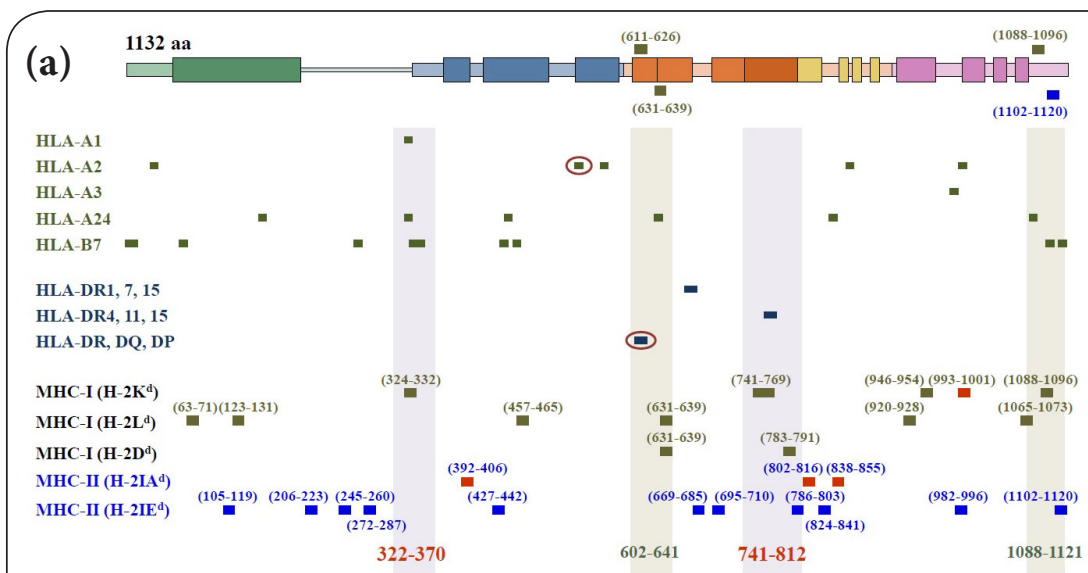

(b) hTERT epitopes (hTERTepi)

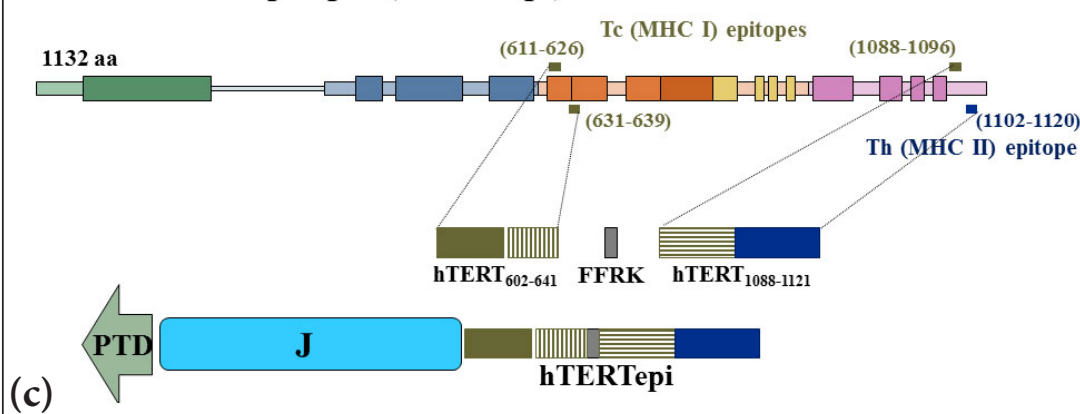

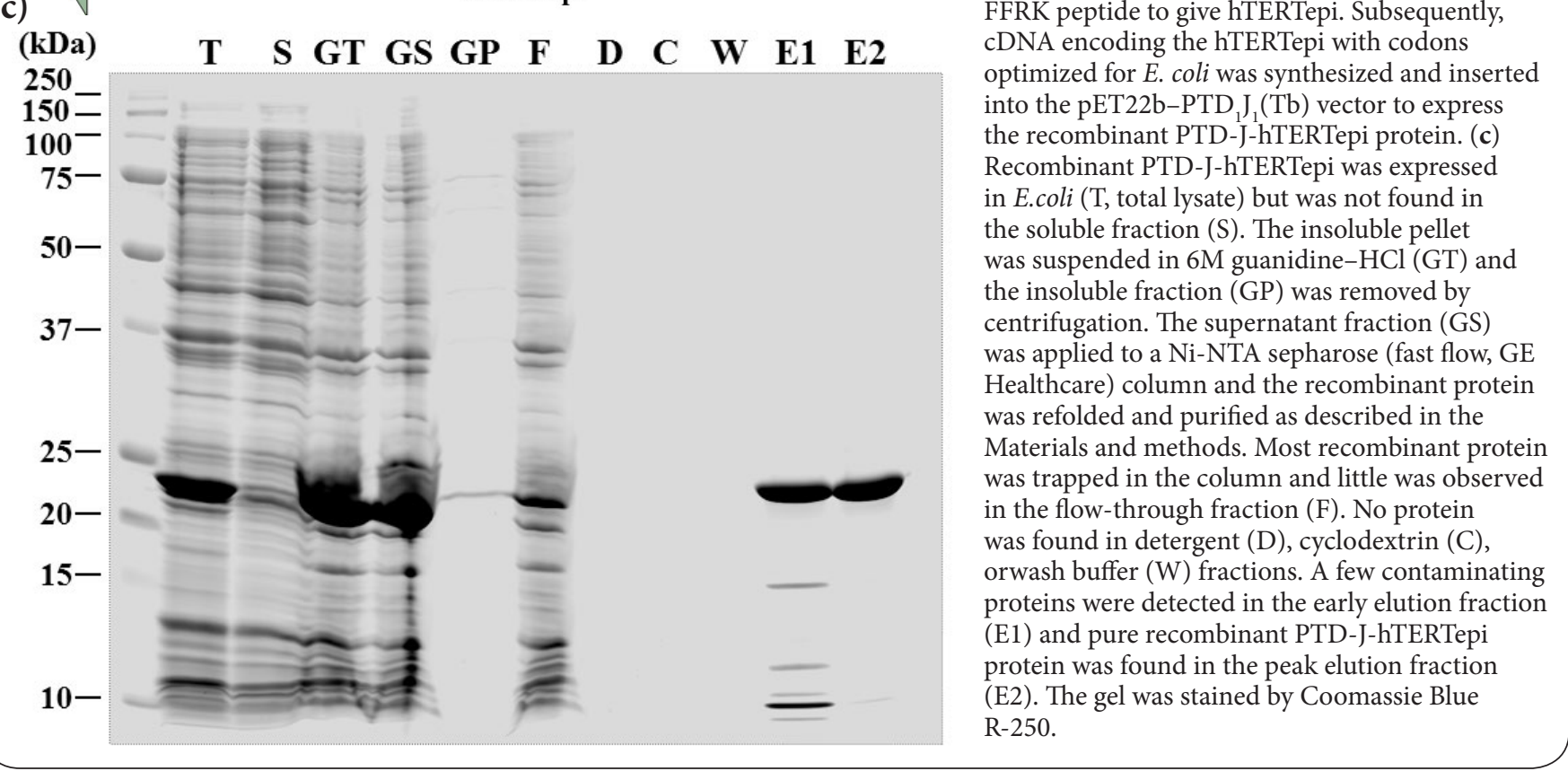

(hTERT\#10) cells as demonstrated in ELISPOT experiments (Figure $2 \mathrm{c}$ ). The major IFNy secreting cells were activated $\mathrm{T}_{H} 1$ and CTL. Recombinant PTD-J-hTERTepi protein also reactivated about 35 of $10^{6}$ splenocytes (Figure $2 \mathrm{c}$ ), indicating that it penetrated actively or passively into splenocytes and was subsequently presented on $\mathrm{MHCI}$ and II molecules, leading to activation of latent memory $T$ cells.
Figure 3. Amino acid sequence analyses, expression vector construction, and recombinant hTERT T-cell epitope expression.

(a) Published human MHC class I (HLA-A1, A2, A3, A24 and B7 allele) and class II (HLA-DR1, DR4, DR7, DR11 and DR15 allele and pan DR, DQ and DP) associated hTERT peptides are summarized. Sequences ofhTERT peptides were predicted using ANN [17] and SMM methods $[18]$ and associations with mouse MHC class I and II of $\mathrm{H}-2^{\mathrm{d}}$ genotype with IC50 values of $<150$ $\mathrm{nM}$ are listed. (b) The peptides hTERT ${ }_{602-641}$ and hTERT $_{1088-1121}$ were selected and linked using an FFRK peptide to give hTERTepi. Subsequently, cDNA encoding the hTERTepi with codons into the $\mathrm{pET} 22 \mathrm{~b}-\mathrm{PTD}_{1} \mathrm{~J}_{1}(\mathrm{~Tb})$ vector to express the recombinant PTD-J-hTERTepi protein. (c) Recombinant PTD-J-hTERTepi was expressed in E.coli (T, total lysate) but was not found in was suspended in $6 \mathrm{M}$ guanidine- $\mathrm{HCl}(\mathrm{GT})$ and the insoluble fraction (GP) was removed by centrifugation. The supernatant fraction (GS) was applied to a Ni-NTA sepharose (fast flow, GE was refolded and purified as described in the Materials and methods. Most recombinant protein in the flow-through fraction (F). No protein was found in detergent (D), cyclodextrin (C), (E1) and pure recombinant PTD-J-hTERTepi protein was found in the peak elution fraction R-250.

\section{PTD-J-hTERTepi suppressed CT26 (hTERT\#10) tumor growth}

Because the hTERTepi polypeptide contained epitopes of hTERT, we examined the efficacy of PTD-J-hTERTepi as a vaccine against CT26 (hTERT\#10) cancer cells. In these experiments, syngeneic DCs were used to augment antigen presentation, and recombinant Hsp72, which associates with the J domain 
Lin et al. Journal of Immunotherapy Applications 2015,

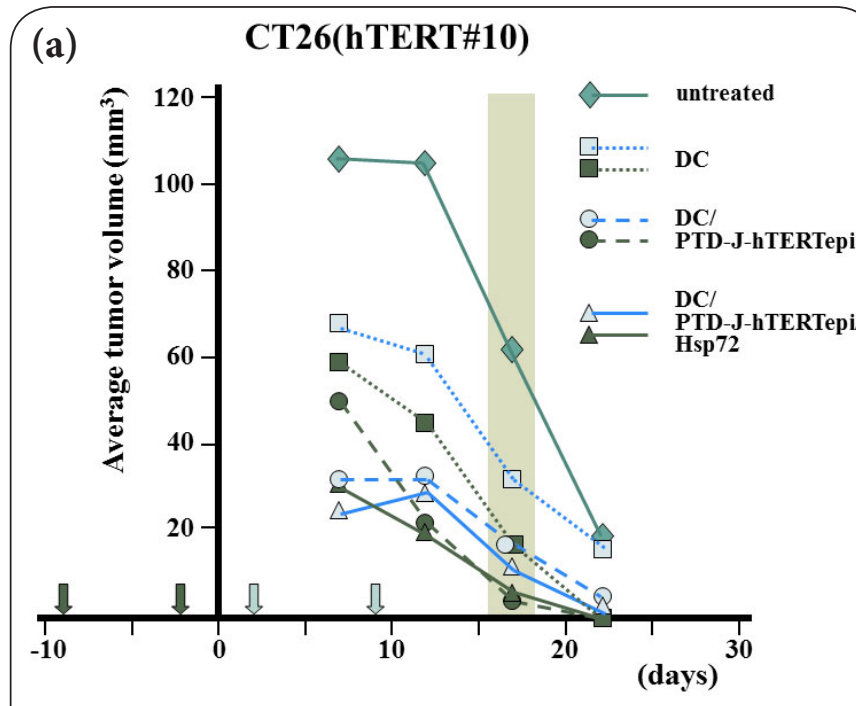

(b)

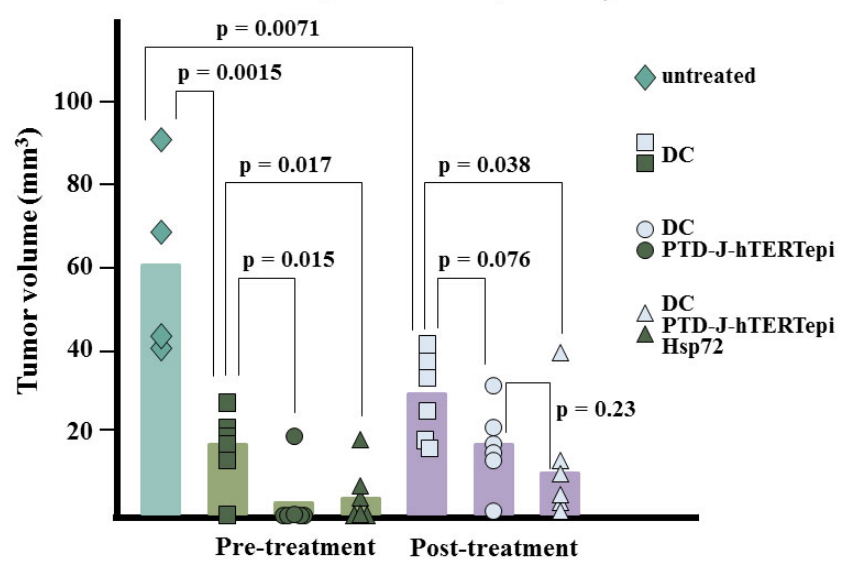

Figure 4. Cellular immunity in the presence of PTD-JhTERTepi.

(a) CT26(hTERT\#10) transplantation was performed (day 0) in two separate sets of mice. The pre-treatment immunization was performed at 9 and 2 days before transplantation, and post-treatment immunization was performed at 2 and 9 days after transplantation, and sets of mice were further divided into three subsets for intraperitoneal injections of DCs alone, DCs and $35 \mu \mathrm{g}$ of PTD-J-hTERTepi, and DCs with $35 \mu \mathrm{g}$ of PTD-J-hTERTepi and $137 \mu \mathrm{g}$ of Hsp72, in $0.5 \mathrm{~mL}$ of PBS.

Tumor volumes were measured every fifth day from day 7 after CT26(hTERT\#10) implantation. (b) Tumor volumes were compared onthe $17^{\text {th }}$ day.

was added. In addition, extra-cellular $\mathrm{Hsp} 72$ also acts as a danger signal [20] and activates innate immunity via Tolllike receptor (TLR) 2 and TLR4 pathways [13,21]. Accordingly, DCs were peritoneally injected alone, with PTD-J-hTERTepi, and with PTD-J-hTERTepi and Hsp72 at9 and 2 days before CT26(hTERT\#10) cell transplantation. As shown in Figure 4a, significant CT26(hTERT\#10) tumor growth was observed in untreated mice until the twelth day after transplantation and subsequently decreased to undetectable volumes by the $27^{\text {th }}$

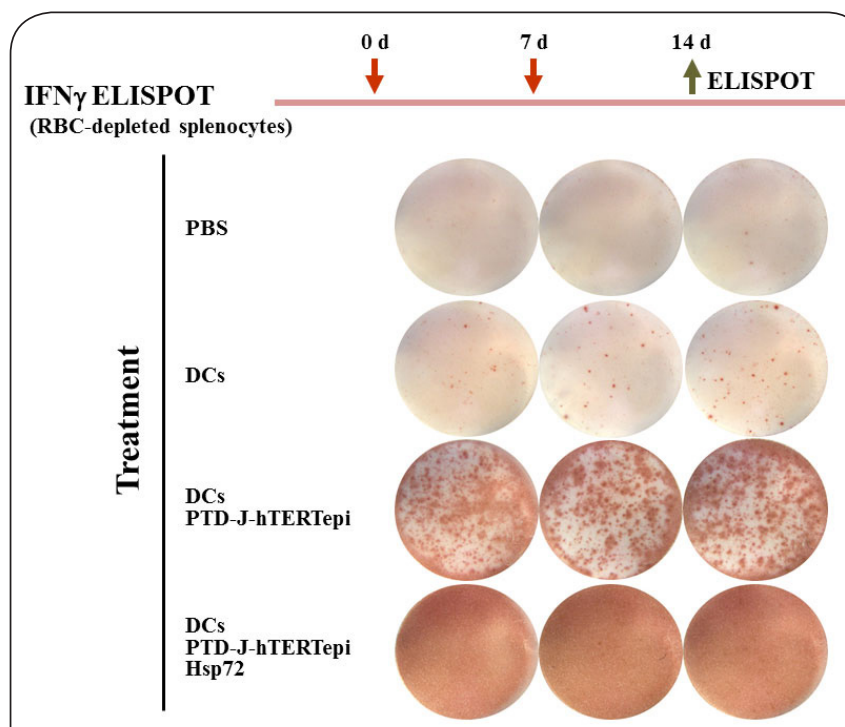

Figure 5. Activation of interferon $\gamma$-expressing splenocytes by PTD-J-hTERTepi and Hsp72.

Four sets of mice received treatments with PBS, $1 \times 10^{6}$ differentiated DCs, DCs with $35 \mu \mathrm{g}$ of PTD-J-hTERTepi, and DCs with $35 \mu \mathrm{g}$ of PTD-J-hTERTepi and $13 \mu \mathrm{g}$ of Hsp72, in a volume of $0.5 \mathrm{~mL}$ on days 0 and 7 . Numbers of IFN $\gamma$ expressing splenocytes were determined at day 14 using ELISPOT. Measurements were performed in triplicate.

day. Tumor sizes were significantly smaller on mice pretreated with DCs, DCs/PTD-J-hTERTepi, and DCs/PTD-J-hTERTepi/Hsp72 than on untreated mice, and receded from the seventh day after transplantation. On the $17^{\text {th }}$ day, significant differences in tumor volumes between treatments demonstrated that injections of DCs suppressed CT26 (hTERT\#10) tumor growth $(\mathrm{p}=0.0015)$, and that co-injection of PTD-J-hTERTepi and PTDJ-hTERTepi with Hsp72 further suppressed CT26 (hTERT\#10) tumor growth ( $p=0.015$ and 0.017 , respectively). The effects of DCs, DCs/PTD-J-hTERTepi and DCs/PTD-J-hTERTepi/Hsp72 on CT26(hTERT\#10) tumor sizes following intraperitoneal injections on days 9 and 2 after transplantation led to similar observations as those after pretreatments. Accordingly, tumor volumes on the $17^{\text {th }}$ day differed significantly between post treatment groups, and indicate suppression of CT26 (hTERT\#10) tumor growth by DCs $(p=0.0071)$ and additional suppression by PTD-J-hTERTepi and PTD-J-hTERTepi with Hsp72 ( $p=0.076$ and 0.038 , respectively). However, the tumor suppressive effect of Hsp72 treatment alone was not significant $(p=0.23)$. Besides the cross presentation of the hTERT epitopes from PTD-J-hTERTepi to the host immune system, the bone marrow derived DCs themselves could suppress CT26 (hTERT\#10) tumor growth (Figure 4a). And the effect of DCs on CT26 (hTERT\#10) tumor suppression was significant (Figure 4b). This phenomenon could be interpreted that the injected DCs enhanced the probability to cross present the hTERT epitopes from CT26 (hTERT\#10) tumors. That is to say, 
Lin et al. Journal of Immunotherapy Applications 2015,

the bone marrow derived DCs of oneself could be used as a tumor suppressing agent.

\section{DCs with PTD-J-hTERTepi stimulate splenocytes to secret IFN $\gamma$}

Suppressive effects of pre- and post-transplantation injections of DCs on CT26 (hTERT\#10) tumor growth likely reflect the consequent induction of host immunity. Accordingly, only 9 of $10^{6}$ splenocytes secreted IFN $\gamma$ at seven days after the second injection of DCs, where as about 510 of $10^{6}$ splenocytes secreted IFN $\gamma$ at seven days in mice treated with DCs/ PTD-J-hTERTepi (Figure 5), suggesting a predominant role of PTD-J-hTERTepi in the induction of IFNY. Previous studies show that recombinant $\mathrm{Hsp} 72$ activates innate immunity via Toll-like receptor TLR2 and TLR4 pathways $[13,21]$ and enhances humoral immunity against PTD-J-recombinant proteins $[12,13]$. In agreement, the present data show that more than 3,000 of $10^{6}$ splenocytes secreted IFN $\gamma$ after treatment with DCs/PTD-J-hTERTepi/Hsp72. Moreover, membrane-bound and exported $\mathrm{Hsp} 70$ reportedly initiated secretion of proinflammatory cytokines [22]. Hence, the dramatic increase in numbers of IFN $\gamma$ secreting cells following treatments with Hsp72 may indicate the presence of a proinflammatory $T_{H} 1$ host response via TLR2 [23]. However, further experiments are needed to characterize this phenomenon.

\section{Additional files}

\section{Supplementary Figure S1}

Supplementary Figure S2

Supplementary Figure S3

\section{Competing interests}

The authors declare that they have no competing interests.

Authors' contributions

\begin{tabular}{|l|c|c|c|}
\hline Authors' contributions & TYL & YHS & CKC \\
\hline Research concept and design & -- & -- & $\checkmark$ \\
\hline Collection and/or assembly of data & $\checkmark$ & $\checkmark$ & -- \\
\hline Data analysis and interpretation & $\checkmark$ & $\checkmark$ & $\checkmark$ \\
\hline Writing the article & $\checkmark$ & -- & $\checkmark$ \\
\hline Critical revision of the article & -- & -- & $\checkmark$ \\
\hline Final approval of article & $\checkmark$ & -- & $\checkmark$ \\
\hline Statistical analysis & $\checkmark$ & -- & -- \\
\hline
\end{tabular}

Acknowledgement and funding

This work was supported by the grants NSC102-2320-B-

059-001-MY3 and NSC 99-2313-B-059-004-MY3 from the

Ministry of Science and Technology, Taiwan to CKC.

Publication history

Editor: Shao-An Xue, University College London Medical School, UK. Received: 15-Oct-2015 Final Revised: 20-Nov-2015

Accepted: 30-Nov-2015 Published: 09-Dec-2015

\section{References}

1. Kim NW, Piatyszek MA, Prowse KR, Harley CB, West MD, Ho PL, Coviello GM, Wright WE, Weinrich SL and Shay JW. Specific association of human telomerase activity with immortal cells and cancer. Science. 1994; 266:2011-5. | Article | PubMed

2. Kim NW. Clinical implications of telomerase in cancer. Eur J Cancer. 1997; 33:781-6. | Article | PubMed

3. Shay JW and Bacchetti S. A survey of telomerase activity in human cancer. Eur J Cancer. 1997; 33:787-91. | Article | PubMed

4. Ruden $\mathrm{M}$ and Puri N. Novel anticancer therapeutics targeting telomerase. Cancer Treat Rev. 2013; 39:444-56. | Article | PubMed

5. Crees Z, Girard J, Rios Z, Botting GM, Harrington K, Shearrow C, Wojdyla L, Stone AL, Uppada SB, Devito JT and Puri N. Oligonucleotides and G-quadruplex stabilizers: targeting telomeres and telomerase in cancer therapy. Curr Pharm Des. 2014; 20:6422-37. | Article | PubMed

6. Vonderheide RH. Universal tumor antigens for cancer vaccination: targeting telomerase for immunoprevention. Discov Med. 2007; 7:1038. | Article | PubMed

7. Beatty $\mathrm{GL}$ and Vonderheide RH. Telomerase as a universal tumor antigen for cancer vaccines. Expert Rev Vaccines. 2008; 7:881-7. | Article | PubMed

8. Liu JP, Chen W, Schwarer AP and Li H. Telomerase in cancer immunotherapy. Biochim Biophys Acta. 2010; 1805:35-42. | Article | PubMed

9. Adotevi O, Dosset M, Galaine J, Beziaud L, Godet $Y$ and Borg C. Targeting antitumor CD4 helper T cells with universal tumor-reactive helper peptides derived from telomerase for cancer vaccine. Hum Vaccin Immunother. 2013; 9:1073-7. | Article | PubMed Abstract | PubMed Full $\underline{\text { Text }}$

10. Ruczynski J, Wierzbicki PM, Kogut-Wierzbicka M, Mucha P, SiedleckaKroplewska K and Rekowski P. Cell-penetrating peptides as a promising tool for delivery of various molecules into the cells. Folia Histochem Cytobiol. 2014; 52:257-69. | Article | PubMed

11. Jiang Y, Li M, Zhang Z, Gong T and Sun X. Cell-penetrating peptides as delivery enhancers for vaccine. Curr Pharm Biotechnol. 2014; 15:256-66. | Article | PubMed

12. Chuang CK, Su YS, Fan CT, Lee WC and Chen MY. A dual-functional E. coli vector for expressing recombinant protein with high solubility and antigen presentation ability. Protein Expr Purif. 2009; 65:51-6. | Article I PubMed

13. Lin TY, Su YS, Lin TY, Hung LY and Chuang Ck. Hsp70 Protein Can Enhance the Immunogenicity of FMDV VP1 Epitopes Fused with PTD-J Domain. Scholarly J. Biol. Sci. 2013; 2:39-48. | Pdf

14. Lin TY, Su YH, Lee KH and Chuang CK. The J-domain of heat shock protein $\mathbf{4 0}$ can enhance the transduction efficiency of arginine-rich cell-penetrating peptides. Biomed Res Int. 2015; 2015:698067. | Article | PubMed Abstract I PubMed Full Text

15. Adotevi O, Mollier K, Neuveut C, Dosset M, Ravel P, Fridman WH, Tartour E, Charneau P, Wain-Hobson S and Langlade-Demoyen P. Targeting human telomerase reverse transcriptase with recombinant lentivector is highly effective to stimulate antitumor CD8 T-cell immunity in vivo. Blood. 2010; 115:3025-32. | Article | PubMed

16. Dosset M, Godet Y, Vauchy C, Beziaud L, Lone YC, Sedlik C, Liard C, Levionnois E, Clerc B, Sandoval F, Daguindau E, Wain-Hobson S, Tartour E, Langlade-Demoyen $\mathrm{P}$, Borg $\mathrm{C}$ and Adotevi $\mathrm{O}$. Universal cancer peptidebased therapeutic vaccine breaks tolerance against telomerase and eradicates established tumor. Clin Cancer Res. 2012; 18:6284-95. | Article I PubMed

17. Nielsen M, Lundegaard C, Worning P, Lauemoller SL, Lamberth K, Buus S, Brunak $S$ and Lund $O$. Reliable prediction of T-cell epitopes using neural networks with novel sequence representations. Protein Sci. 2003; 12:1007-17. | Article | PubMed Abstract | PubMed Full Text

18. Peters $B$ and Sette A. Generating quantitative models describing the sequence specificity of biological processes with the stabilized matrix method. BMC Bioinformatics. 2005; 6:132. | Article | PubMed Abstract | PubMed Full Text

19. Harada Y, Ueda Y, Kinoh H, Komaru A, Fuji-Ogawa T, Furuya A, lida A, Hasegawa M, Ichikawa T and Yonemitsu Y. Cytokine-based log-scale expansion of functional murine dendritic cells. PLoS One. 2009; 4:e6674. | Article | PubMed Abstract | PubMed Full Text 
Lin et al. Journal of Immunotherapy Applications 2015,

http://www.hoajonline.com/journals/pdf/2055-2394-2-1.pdf

doi: 10.7243/2055-2394-2-1

20. Campisi J, Leem TH and Fleshner M. Stress-induced extracellular Hsp72 is a functionally significant danger signal to the immune system. Cell Stress Chaperones. 2003; 8:272-86. | PubMed Abstract | PubMed Full $\underline{\text { Text }}$

21. Asea A, Rehli M, Kabingu E, Boch JA, Bare O, Auron PE, Stevenson MA and Calderwood SK. Novel signal transduction pathway utilized by extracellular HSP70: role of toll-like receptor (TLR) 2 and TLR4. J Biol Chem. 2002; 277:15028-34. | Article | PubMed

22. Radons J and Multhoff $\mathrm{G}$. Immunostimulatory functions of membranebound and exported heat shock protein 70. Exerc Immunol Rev. 2005; 11:17-33. | Pdf | PubMed

23. Murciano C, Yanez A, Gil ML and Gozalbo D. Both viable and killed Candida albicans cells induce in vitro production of TNF-alpha and IFN-gamma in murine cells through a TLR2-dependent signalling. Eur Cytokine Netw. 2007; 18:38-43. | Article | PubMed

\section{Citation:}

Lin T-Y, Su Y-H and Chuang C-k. PTD-J domain acts as an antigen carrier for cell mediated immunity. $J$ Immunother Appl. 2015; 2:1.

http://dx.doi.org/10.7243/2055-2394-2-1 